GSA Data Repository Item 2015203

\title{
Table DR1
}

long lat -err_phi phi +err_phi -err_dt dt +err_dt stn rating phase date lat long depth Mw method -64.33 18.73 -86 -52.9 -26.6 0.4 0.8 1.3 ABVI fair SKS 3/9/201138.4 142.8 32 7.3 RC $-64.3318 .7379 .9-88.5-77.922 .22 .4$ ABVI fair SKS 7/31/2011 -17 171.6 106.1 RC $-64.3318 .73-84-57.8-31.71 .21 .82 .3$ ABVI fair SKS 6/19/2012 53.4 171.6 14 6 RC -64.33 18.73 -83 -66.5 -20.6 0.7 1.8 2.6 ABVI fair SKS 6/24/2012 57.6 163.2106 RC $-64.3318 .73-31.7-16.2-2.50 .10 .81 .4$ ABVI fair SKS 6/29/2012 43.4 84.7 18 6.3 RC $-64.3318 .7344 .756 .169 .90 .81 .01 .2 \mathrm{ABVI}$ fair SKKS 8/12/2012 35.7 82.5 13 6.3 RC $-64.3318 .73-88-31.45 .500 .43 .1$ ABVI fair SKKS 8/14/2012 49.8 145.1583 7.7 RC $-64.3318 .7380 .9-73.5-55.811 .31 .6$ ABVI fair SKKS 9/5/2012 -12.5 166.5 276 RC $-64.3318 .7388-53.310 .60 .61 .12$ ABVI fair SKKS 10/9/2012 -60.3153.7 10 6.6 RC -64.3318 .7337 .763 .6880 .4 0.6 0.9 ABVI fair SKKS 1/28/2013 42.6 79.7 15 6.2 RC $-64.3318 .73-83-48.5-19.60 .31 .01 .9$ ABVI fair SKKS 1/1/2014 -13.9 167.2 196 6.5 RC -64.71 17.73 55.8 84.9 -71.9 0.4 0.9 1.7 CDVI fair SKKS 2/12/201435.9 82.510 6.9 RC $-64.7117 .7337-63.02-36$ 0.2 0.6 2.4 CDVI fair SKS 6/5/2012 34.94 141.13 15 6.1 RC $-64.7117 .73-237.00320 .30 .81 .4$ CDVI fair SKS 1/9/2008 32.29 85.17 10 6.4 RC $-64.7117 .7382-67.0-31$ 0.1 0.5 1.8 CDVI fair SKS 12/7/2012 37.89 144.0936 7.2 RC $-64.7117 .7383-76.0-52.8$ 0.2 0.6 2.7 CDVI fair SKS 7/10/201138143.3 237 RC -64.71 17.73 333.5650 .00 .35 1.2 CDVI fair SKS 3/31/2006 -29.61-176.82 17 6.5 RC -65.86 18.27 -88 -66.0 -46 0.2 0.4 0.7 CBYP fair SKS 6/14/2005 51.23 179.41516.8 RC -65.86 18.27 -83 -57.0 -34 0.0 0.2 0.5 CBYP fair SKS 3/31/2006 33.5 38.78 7 6.1 RC $-65.8618 .27-55-25.030 .2$ 0.6 1.1 CBYP fair SKS 11/24/2008 54.2 154.3 492 7.3 RC $-65.8618 .27-55-27.0-2$ 0.1 0.4 0.9 CBYP fair SKS 5/23/2013 54.87 153.28 609 8.3 RC $-65.8618 .27-44-29.0-160.60 .81 .0$ CBYP fair SKS 11/12/2013 54.68 162.29 47 6.5 RC $-65.8618 .27-43-18.060 .2$ 0.6 1.1 CBYP fair SKS 10/1/2013 53.17 152.77578 6.7 RC -65.86 18.27 -44.7 -22.0 -0.5 0.2 0.7 1.3 CBYP fair SKS 5/11/2013 -17.9 -175.1 2056.4 RC -65.86 18.27 -65 -42.0 -22 0.0 0.4 0.9 CBYP fair SKS 2/26/2006 -23.61 -179.99 5356.4 RC $-66.7518 .35-56.8-19.0-3.5$ 0.8 1.9 2.6 AOPR fair SKKS 6/30/2010 -23.3 179.1 581 6.4 RC $-66.7518 .3571 .9-50.2-22.6$ 0.6 1.2 3.3 AOPR fair SKS 8/10/2010 -17.5 168.1 25 7.3 RC $-66.7518 .3589-55.6-16.60 .20 .7$ 1.8 AOPR fair SKS 8/16/2010 -20.8 -178.8 6036.2 RC $-66.7518 .35-53.8-26.9-2.5$ 1.2 1.8 2.4 AOPR fair SKS 12/29/2010 -19.7 168.1 16 6.3 RC $-66.7518 .35-67.9-37.1-7.5$ 0.7 1.0 1.5 AOPR fair SKKS 1/13/2011 -20.6 168.5 9.9 RC $-66.7518 .3570 .9-69.9-36.7$ 0.8 1.3 2.2 AOPR fair SKKS 3/9/201138.3 142.8 22 6.5 RC -66.75 18.35 67.9 -82.5 -36.7 0.8 1.9 2.2 AOPR fair SKS 3/13/201135.7 141.6 8 6.2 RC $-66.7518 .35-8.55 .1019 .61 .62 .02 .4$ AOPR fair SKKS 3/15/201137.6 142.2 286.1 RC $-66.7518 .3550 .8-75.0-5.6$ 0.6 1.1 2.1 AOPR fair SKKS 3/15/201140.3143.3 206 RC $-66.7518 .3586-74.9-51.81 .3$ 2.1 2.7 AOPR fair SKKS 3/25/2011 38.8 141.9 39 6.2 RC -66.75 18.35 88 -65.2 -33.7 0 0.5 1.2 AOPR fair SKS 3/27/201138.414219 RC $-66.7518 .35-76.9-45.2-16.6$ 0.4 0.8 1.2 AOPR fair SKS 4/3/2011 -17.6 -178.6 5526.4 RC $-66.7518 .3570 .9-82.5-51.8$ 0.6 1.1 1.7 AOPR fair SKKS 4/21/2011 35.6 140.3 43 6.2 RC 
GSA Data Repository Item 2015203

-66.75 18.35 32.7 -77.4 -32.7 0.3 1.1 3.1 AOPR fair SKS 6/24/201257.6 163.2 10 6 RC $-66.7518 .35-57.8-42.3-33.7$ 1.6 2.2 2.7 AOPR fair SKS 4/26/2013 -28.7 -178.9 351 6.2 RC -66.75 18.35 66.9 -72.9 -37.7 0.6 1.0 1.8 AOPR fair SKS 5/23/2013 -20.6 -175.7 154 6.3 RC -66.75 18.35 61.8 77.0 -61.8 0.7 1.3 1.8 AOPR fair SKS 6/15/2013 -33.9 179.5 172 6 RC -66.75 18.35 76.9 -86.4 -68.9 1 1.4 1.9 AOPR fair SKS 8/12/2013 -30.6 -179.6 3256.1 RC -66.75 18.35 56.8 67.7 -90 11.5 1.9 AOPR fair SKS 8/16/2013 -41.8 174.1 10 6.5 RC -66.75 18.35 33.7 -79.1 -46.8 0.1 0.7 3.2 AOPR fair SKS 8/282013 -27.8 179.7489 6.2 RC -63.07 18.05 60.8 -85.3 -53.8 0.9 1.32 SMRT fair SKS 7/23/2008 39.8 141.5 108 6.8 RC -63.07 18.05 46.8 72.6 -85 0.9 1.3 2.1 SMRT fair SKS 8/4/2010 51.4 -178.7 27 6.4 RC -63.24 17.62 85 -68.1 -39.7 0.2 0.6 1.3 SABA fair SKS 2/18/2009 -27.4 -176.3 257 RC -63.24 17.62 -82 -52.9 -26.6 0.2 0.8 1.4 SABA fair SKS 4/7/2009 46151.6316 .9 RC -63.24 17.62 -55.8 -42.2 26.6 1.2 1.7 2.1 SABA fair SKS 4/26/2009 -30.3 -178.6 132 6.1 RC -63.24 17.62 74.9 -89.7 -71.9 1.6 2.0 2.5 SABA fair SKS 2/18/2010 42.6 130.7578 6.9 RC -63.24 17.62 58.8 69.3 -90 1.2 2.0 2.6 SABA fair SKKS 3/14/2010 37.7 141.6 32 6.5 RC -63.24 17.62 79.9 -74.7 -17.6 0.4 0.8 2.1 SABA fair SKS 11/3/2010 -20.5 -174.3 19 6.1 RC -63.24 17.62 -90 -65.8 -38.7 1.3 1.8 2.3 SABA fair SKS 2/20/201155.9 162.1336.2 RC -63.24 17.62 -86 -64.4 -42.7 0.6 0.9 1.3 SABA fair SKS 9/26/2012 51.6 -178.3 5786.7 RC -63.24 17.62 74.9 -63.8 -36.7 0.6 1.1 2.1 SABA fair SKS 11/12/2013 54.7 162.3476.5 RC -61.79 17.67 80 -71 -48 1.1 1.8 3.0 ANWB fair SKS 4/19/2013 46.18 150.8122 7.3 RC -61.79 17.67 81-68 -31 0.3 1.1 3.1 ANWB fair SKS 7/24/201257.6 163.2 17 6.1 RC $-61.7917 .67-88-68-50.80 .71 .01 .5$ ANWB fair SKS 7/23/2008 39.8 141.5 108 6.8 RC $-61.7917 .6783-75-490.81 .2$ 2.1 ANWB fair SKKS 10/16/2007 -25.51 179.5 477 6.6 RC -61.79 17.67 55.8 73.1 -71.9 0.7 1.3 1.8 ANWB fair SKS 12/21/2007 51.4 -179 30 6.3 RC -61.79 17.67 -77.9 -52.3 -34.7 0.6 1.1 1.8 ANWB fair SKKS 1/15/2008 -22 -179.5 598 6.5 RC -61.79 17.67 51.8 81.2 -68.9 0.6 1.2 2.3 ANWB fair SKS 3/3/2008 46.4 153.210 6.5 RC -61.79 17.67 78.9 -76.4 -47.8 0 0.7 1.5 ANWB fair SKS 4/16/2008 51.9 -179.2 13 6.6 RC -61.79 17.67 -79.9 -58.4 -37.7 0.6 1.0 1.6 ANWB fair SKS 7/19/2008 -17.3 -177.3 391 6.4 RC -61.79 17.67 58.8 80.7-64.9 1.7 2.6 3.6 ANWB fair SKS 7/23/2008 39.8 141.5 1086.8 RC -61.79 17.67 47.8 -75.0 -47.8 0.4 1.14 ANWB fair SKS 10/5/2008 -30.2 -177.210 6.1 RC -61.79 17.67 -79.9 -61.9 -43.7 0.4 0.8 1.2 ANWB fair SKS 2/18/2009 -27.4 -176.3 25 7 RC -61.79 17.67-47.8 -29.5 -12.6 0.6 0.9 1.4 ANWB fair SKS 2/18/2010 42.6 130.7578 6.9 RC $-61.7917 .67-82-63-460.20 .81 .2$ ANWB fair SKS 9/30/2013 -30.88 -178.38 42 6.4 RC -62.98 17.49-82 -52.1 -15.6 0.4 0.9 2.4 SEUS fair SKS 2/18/2009 -27.4 -176.3 25 7 RC $-62.9817 .49-74.9-51.9-30.7$ 0.1 0.7 1.2 SEUS fair SKS 5/11/2013 -17.9 -175.1 205 6.4 RC -62.98 17.49-65.9-49.4 -33.7 11.2 1.6 SEUS fair SKS 5/24/13 54.9 153.3609 8.3 RC -62.98 17.49 79.9 -74.7 -43.7 0.5 1.1 1.8 SEUS fair SKS 11/12/2013 54.7 162.3 47 6.5 RC $-64.9618 .35-80.9-49.1-13.60 .41 .22 .6$ STVI fair SKS 4/29/2006 60.5 167.5 11 6.6 RC -64.96 18.35-66.9 -50.2 -19.6 0.7 1.1 1.6 STVI fair SKS 11/24/2008 54.2 154.3492 7.3 RC -64.96 18.35 70.9 -89.5 -61.8 0.7 1.1 1.6 STVI fair SKS 3/9/201138.3 142.8 22 6.5 RC -64.96 18.35 $82-68.8-46.80 .81 .4$ 2.8 STVI fair SKS 4/27/201138.4142196.2 RC 
GSA Data Repository Item 2015203

-64.96 18.35 -75.9-46.1 -21.6 1 1.5 2.1 STVI fair SKKS 6/22/201140142.2 33 6.7 RC $-64.9618 .35-86-54-32.7$ 0.5 1.0 1.5 STVI fair SKS 4/9/2013 28.4 51.6 12 6.3 RC $-64.9618 .35-91-76-16$ 0.5 3.0 4.0 STVI fair SKS 4/19/2013 46.18 150.8122 7.3 RC $-64.9618 .35-72.9-48.9-21.60 .40 .7$ 1.2 STVI fair SKS 5/11/2013 -17.9 -175.1 2056.4 RC $-64.9618 .35-68.9-58.0-39.711 .41 .7$ STVI fair SKS 11/12/2013 54.7 162.3 47 6.5 RC $-65.5518 .106877-921.42 .43 .7$ MTP fair SKS 6/14/2005 51.2 179.4 51 6.8 RC -65.55 18.1078 84 -90 1.2 2.1 3.1 MTP fair SKS 10/1/2013 53.2 152.8 578 6.7 RC $-65.5518 .107682-901.72 .8$ 4.0 MTP fair SKS 10/11/2013 -30.75 -178.4 1466.2 RC $-66.1518 .11-$ Inf -89 Inf 0.0 0.9 Inf SJG fair SKS 4/26/2009 -30.3 -179.6 132 6.1 SC $-66.1518 .1170-83-540.40 .51 .0$ SJG fair SKS 3/22/2008 52.2178 .71326 .2 SC $-66.1518 .1172-62-48$ 0.3 0.4 1.0 SJG fair SKS 4/29/2007 $52.0-179.91176 .2$ SC $-66.6118 .045175-850.81 .11 .8$ OBIP fair SKS 2/22/2006 -21.32 33.58 11 7.0 RC $-66.6118 .046787-690.91 .41 .8$ OBIP fair SKS 5/22/2006 60.77 165.74 16 6.6 RC $-66.6118 .043086-670.21 .04 .0$ OBIP fair SKS 7/8/2006 51.2 -179.3 226.6 RC -66.61 18.04 -91 -67 -43 0.2 0.5 0.8 OBIP fair SKS 12/9/2007 -26.0 -177.5 152 7.8 RC $-66.6118 .0467-79-460.10 .81 .6$ OBIP fair SKS 8/4/2010 51.4 -178.7 27 6.4 RC $-66.6118 .04-85-66-500.40 .91 .4$ OBIP fair SKS 1/13/2007 46.2 154.510 8.1 RC -66.6118 .048083881 .11 .41 .7 OBIP fair SKS 8/14/2012 49.8 145.0 583 7.7 RC $-66.6118 .0486-73-530.71 .21 .8$ OBIP fair SKS 11/16/2012 49.3 155.4 29 6.5 RC -67.04 17.97 66 -71 80 1.2 1.7 2.2 MLPR fair SKS 4/9/2013 28.4 51.6 12 6.3 SC -67.04 17.97 82 86 -90 1.5 2.0 2.5 MLPR fair SKS 5/24/2013 54.9 153.3 609 8.3 SC $-67.0417 .97-87-57-290.61 .01 .6$ MLPR fair SKS 11/12/2013 54.7 162.3 47 6.5 SC $-67.0918 .017482-901.41 .9$ 2.5 MGP fair SKS 5/1/2003 39.0 40.5 10 6.3 SC $-67.0918 .017686-840.81 .11 .5$ MGP fair SKS 6/23/2003 51.4 176.8 20 6.9 SC -67.0918 .017479861 .6 2.6 3.6 MGP fair SKS 6/10/2004 55.7 160.01896.8 SC -67.14 18.21 -82 -70 -62 1.6 2.1 2.7 MPR fair SKS 2/2/2006 -17.8 -178.4 5986.7 SC $-67.1418 .2184-65-23$ 0.3 1.1 3.3 MPR fair SKS 6/9/2006 -17.5 -178.8 5646.1 SC -67.14 18.21 70 80 -82 1.1 1.9 3.0 MPR fair SKS 7/8/2006 51.2 -179.322 6.6 SC $-67.1118 .477280-801.23 .34 .0$ AGPR fair SKS 5/30/2007 52.1 157.3116 6.4 SC $-67.1118 .4782-75-390.3$ 0.5 1.3 AGPR fair SKS 10/5/2007 -25.2 179.5 509 6.5 SC -67.11 18.47 74 86 -68 0.5 1.3 2.1 AGPR fair SKS 3/18/2008 -29.3 -177.4 25 6.2 SC $-67.1118 .476882-660.51 .01 .9$ AGPR fair SKS 8/4/2010 51.4 -178.7 276.4 SC -67.11 18.47 -Inf -88 Inf 0.0 1.1 Inf AGPR fair SKS 2/21/2011 -26.1 178.4 5586.5 SC -66.26 18.48 -71 -47 -24 0.8 1.1 1.5 EMPR fair SKS 5/11/2013 -17.9 -175.1205 6.4 SC $-66.2618 .487089-460.30 .8$ 2.0 EMPR fair SKS 7/15/2013 -33.9 179.5 1726 SC -66.2618 .486064702 .3 3.7 4.0 EMPR fair SKS 7/21/2013-41.7 174.4146.5 SC $-68.3818 .51-88-67-460.7$ 1.0 1.5 PCDR fair SKS 5/11/2013 - $17.9-175.12056 .4$ SC $-64.3318 .73-83-66.5-46.8$ 0.9 1.1 1.4 ABVI good SKS 4/3/2011 -17.6 -179.6 552 6.4 RC $-64.3318 .73-86-67.0-42.71 .21 .92 .5$ ABVI good SKS 4/7/2011 38.3 141.6 42 7.1 RC $-64.3318 .73-90-65.7-41.71 .8$ 2.2 2.7 ABVI good SKS 4/11/201135.4 140.6 15 6.2 RC 
GSA Data Repository Item 2015203

-64.33 18.73 89 -75.2 -57.8 11.4 1.7 ABVI good SKS 4/18/2011 -34.3 179.9 86 6.5 RC -64.33 18.73 -72.9 -59.5 -46.8 1.7 2.0 2.3 ABVI good SKS 7/6/2012 35.7 82.5 13 6.2 RC -64.3318 .7388 -76.8 -57.8 1.1 1.4 1.7 ABVI good SKKS 9/26/2012 51.6 -178.3 16 6.5 RC -64.33 18.73 -78.9 -70.2 -63.9 2.8 3.5 4 ABVI good SKKS 11/11/2012 2395.9146 .9 RC -64.33 18.73 -69.9 -52.5 -35.7 1.4 1.8 2.1 ABVI good SKKS 11/16/2012 49.3 155.4 29 6.5 RC -66.75 18.35 66.9 -72.5 -11.6 0.7 1.1 1.7 AOPR good SKS 1/31/2011 -22 -175.6 766 RC $-66.7518 .35-66.9-34.1-16.60 .81 .11 .7$ AOPR good SKS 2/21/2011 -26.1 178.4 558 6.5 RC -66.75 18.35 -78.9 -57.6 -38.7 0.5 0.9 1.4 AOPR good SKS 7/29/2011 57.6 163.2 10 6 RC -66.75 18.35 -85 -63.3 -40.7 0.6 0.9 1.3 AOPR good SKS 5/11/2013 -17.9 -175.1 2056.4 RC -66.75 18.35 78.9 -73.1 -41.7 0.6 1.4 0.8 AOPR good SKS 5/24/2013 54.9 153.3609 8.3 RC -66.75 18.35 77.9 -83.9 -55.8 0.8 1.1 1.5 AOPR good SKS 5/24/201352.2151.5 623 6.7 RC $-66.7518 .3586-74.8-56.8$ 0.4 0.7 1 AOPR good SKS 9/30/2013 -30.9 -178.4 42 6.4 RC -66.75 18.35 80.9 -73.0 -44.7 0.4 0.7 1.2 AOPR good SKS 10/1/2013 53.2 152.8 578 6.7 RC -66.75 18.35 74.9-78.6 56.8 0.6 1.0 1.6 AOPR good SKS 10/11/2013-30.8 -178.4 146 6.2 RC -64.71 17.73 77.9 -72.3 -45.8 1.1 1.6 1.8 CDVI good SKS 11/4/201137 140.4 11 6.7 RC -64.71 17.73 -90 -69.3-48.8 11.5 1.8 CDVI good SKS 11/3/201135.4140.6 15 6.2 RC -64.71 17.73 -72 -60.7 -54 1.4 1.9 2.4 CDVI good SKKS 7/19/2011 40.09 71.41 20 6.2 RC $-64.7117 .7388-48.020 .51 .02 .2$ CDVI good SKS 2/2/2014 -32.91 -177.82 506.4 RC -63.07 18.05-86-61.7-45.8 0.7 1.1 1.6 SMRT good SKS 8/14/2012 49.8 145.1583 7.7 RC -63.07 18.05 83 -79.4 -58.8 0.9 1.1 1.4 SMRT good SKS 5/24/201354.9 153.3609 8.3 RC -63.07 18.05 65.9 83.8 -63.9 0.8 1.2 1.8 SMRT good SKS 10/1/201353.2152.8578 6.7 RC -63.24 17.62 58.8 -66.4 -16.6 0.7 1.2 2.2 SABA good SKS 11/24/2008 54.2 154.3 492 7.3 RC -63.24 17.62 87 -64.5 -34.7 0.9 1.2 1.7 SABA good SKS 8/4/2010 51.4 -178.7 27 6.4 RC -63.24 17.62 -77.9 -59.9 -39.7 0.8 1.2 1.7 SABA good SKS 8/14/2012 49.8 145.1583 7.7 RC -63.24 17.62 -90 -66.3 -40.7 0.7 1.1 1.4 SABA good SKS 10/1/201353.2 152.8 578 6.7 RC -61.79 17.67 -87 -58 -38 1.0 1.4 2.1 ANWB good SKS 10/1/2013 53.2 152.8578 6.7 RC -61.79 17.67 87 -43.6 -27.7 0.8 1.9 2.5 ANWB good SKKS 10/16/2007 -25.8 179.5 509 6.6 RC -61.79 17.67 -53.8 -39.5 -21.6 1.8 2.1 2.4 ANWB good SKKS 11/19/2007-21.2 -178.8 558 6.3 RC $-61.7917 .67-86-61.2-36.7$ 0.6 0.9 1.4 ANWB good SKS 12/9/2007 -26 -177.5 152 7.8 RC $-61.7917 .6788-74.3-57.8$ 1.1 1.5 1.9 ANWB good SKS 3/22/2008 52.2 -178.7 132 6.2 RC -61.79 17.67 83 -79.0 -60.8 11.4 1.9 ANWB good SKS 5/2/2008 51.9 -177.5 14 6.6 RC -61.79 17.67-88 -68.5 -50.8 0.7 1.0 1.5 ANWB good SKS 9/29/2008 -29.8 -177.7 36 7 RC -61.79 17.67 -84 -70.7 -59.8 0.5 0.8 1 ANWB good SKS 11/24/2008 54.2 154.3 492 7.3 RC -61.79 17.67 -28.7 -5.6 15.6 0.1 0.4 0.7 ANWB good SKS 1/18/2011 28.8 63.9 68 7.2 RC $-61.7917 .67-90-68-47$ 0.5 1.0 1.7 ANWB good SKS 5/24/2013 54.87 153.28 609 8.3 RC -62.98 17.49 -90 -61.3 -32.7 0.8 1.1 1.5 SEUS good SKS 11/24/2008 54.2 154.3492 7.3 RC -62.98 17.49 85 -69.4 -40.7 0.5 0.8 1.4 SEUS good SKS 8/4/2010 51.4 -178.7 27 6.4 RC -62.98 17.49-87 -64.7-43.7 0.6 0.9 1.2 SEUS good SKKS 8/14/2012 49.8 145.1 583 7.7 RC -62.98 17.49 -66.9 -46.2 -30.711.3 1.7 SEUS good SKS 10/1/2013 53.2 152.8 578 6.7 RC -64.96 18.35 -86 -78.3 -65.9 1.4 2.1 2.6 STVI good SKS 5/24/201354.9 153.3609 8.3 RC 
GSA Data Repository Item 2015203

-65.55 18.10 74 77 -84 2.6 3.0 3.7 MTP good SKS 6/14/2006 51.9 177.1 37 6.5 RC $-65.5518 .1086-86-801.21 .51 .7$ MTP good SKS 4/29/2007 52.0 -179.9 117 6.2 RC -65.55 18.10 82 84 -90 1.5 2.0 2.4 MTP good SKS 11/12/2013 54.7 162.347 6.5 RC $-66.1518 .117281-600.30 .81 .6$ SJG good SKS 5/16/2009-31.5 -178.8 55 6.5 SC -66.15 18.11 74 79-88 0.8 1.6 2.5 SJG good SKS 10/5/2008 -30.2 -177.2 10 6.1 SC -66.15 18.11787986 1.1 1.6 2.1 SJG good SKS 11/24/2008 54.2 154.3492 7.3 SC -66.15 18.1178 85-86 1.0 2.0 3.3 SJG good SKS 12/9/2007-26.0 -177.5 152 7.8 SC $-66.1518 .118086-80$ 0.6 1.1 1.8 SJG good SKS 10/16/2007 -25.8 179.5 509 6.6 SC $-66.1518 .118487-881.42 .02 .7$ SJG good SKS 10/5/2007 -25.2 179.5 509 6.5 SC -66.15 18.11 84 88 -52 0.3 1.5 2.7 SJG good SKS 8/26/2007-17.5 -174.4 127 6.1 SC -65.8518 .146070801 .11 .31 .6 HUMP good SKKS 7/19/2011 40.0 71.4 20 6.2 SC -65.85 18.14 80 85 -90 1.2 1.8 2.4 HUMP good SKS 11/24/2008 54.2 154.3 492 7.3 SC -65.85 18.14 74 82 -92 1.3 1.7 2.2 HUMP good SKS 7/3/2012 -40.0 174.8 2306.3 SC -65.85 18.14 80 84 -92 2.3 3.2 4.0 HUMP good SKS 9/30/2013 -30.9 -178.4 42 6.4 SC $-65.8518 .146678-901.12 .0$ 3.0 HUMP good SKS 7/21/2013 -41.7 174.4 14 6.5 SC -65.85 18.1478 80 86 2.0 3.0 4.0 HUMP good SKS 5/24/201354.9 153.3609 8.3 SC -65.85 18.1480 83 86 2.8 3.3 3.8 HUMP good SKS 4/26/2013 -28.7-178.9 351 6.2 SC -65.85 18.14 80 82 -92 1.2 1.6 2.1 HUMP good SKS 11/12/201354.7162.3476.5 SC -65.8518.14748088 1.2 2.0 2.9 HUMP good SKS 6/24/201257.6 163.2106 SC -65.85 18.14 6881 -80 1.2 2.0 3.1 HUMP good SKS 7/3/2012 -39.9 173.7 236 6.3 SC -65.85 18.14 74 81 -90 1.1 2.4 3.9 HUMP good SKS 10/1/2013 53.2 152.8578 6.7 SC -66.61 18.04 82 -82 -52 0.6 1.1 1.9 OBIP good SKS 4/21/2006 60.5 165.89 6.1 RC -66.61 18.04-90 -75 -57 1.1 1.4 1.7 OBIP good SKS 5/16/2006 -31.8 -179.3 152 7.4 RC $-66.6118 .047686-700.81 .32 .1$ OBIP good SKS 4/19/2013 46.2 150.8 110 7.3 RC -66.61 18.04 82 86 -92 1.2 1.5 1.8 OBIP good SKS 5/24/201354.9 153.3609 8.3 RC $-66.6118 .0470-84-520.51 .22 .9$ OBIP good SKS 4/19/2013 46.2 150.8 122 7.3 RC 66.6118 .048487 -90 1.1 1.4 1.7 OBIP good SKS 10/1/2013 53.2152.8578 6.7 RC $-66.6118 .0486-83-820.91 .11 .4$ OBIP good SKS 11/12/2013 54.7 162.3476.5 RC -67.14 18.21 -90 -73 -58 0.8 1.2 1.7 MPR good SKS 5/22/2006 60.8 165.914 6.6 SC -67.14 18.21 -74 -61 -58 0.9 1.2 1.5 MPR good SKS 6/14/2005 51.2 179.4 51 6.8 SC -67.14 18.21 $84-81$-68 0.8 1.1 1.4 MPR good SKS 4/29/2007 52.0 -179.9 117 6.2 SC -67.14 18.21 84 89-90 2.2 2.5 2.8 MPR good SKS 8/15/2007 50.3 -177.5 96.5 SC -67.14 18.21 -74-61 -58 1.1 1.4 1.6 MPR good SKS 6/15/2013 -33.9 179.5 172 6 SC -67.11 18.47-74-61 -58 0.9 1.2 1.5 AGPR good SKS 6/14/2005 51.2 179.4 51 6.8 SC $-67.1118 .476883-700.50 .81 .5$ AGPR good SKS 4/29/2007 52.0 -179.9 117 6.2 SC -66.26 18.48 -90 -77 -68 0.9 1.1 1.3 EMPR good SKS 4/26/2013 -28.68 -178.9 3516.2 SC $-66.2618 .48-6468722.02 .4$ 3.0 EMPR good SKS 8/16/2013-41.8 174.1 10 6.5 SC -63.38 18.51 -92 -85 -80 1.3 1.6 1.9 PCDR good SKS 5/24/2013 54.9 153.3 609 8.3 SC -63.38 18.51 -92 -82 -83 1.7 1.9 2.1 PCDR good SKKS 9/4/2013 30.0138.8 407 6.5 SC -63.38 18.51-78-62 -52 0.9 1.2 1.5 PCDR good SKKS 9/30/2013 -30.9 -178.4 42 6.4 SC 
GSA Data Repository Item 2015203

-68.38 18.51 $86-85$ 1.1 1.5 2.0 PCDR good SKS 4/19/2013 46.2 150.8 110 7.3 SC

-68.38 18.51 -90 -80 -72 1.2 1.6 1.9 PCDR good SKS 10/1/2013 53.2152.8 578 6.7 SC

-68.38 18.51-88 -79 -76 1.2 1.5 1.7 PCDR good SKS 11/12/2013 54.7 162.3476.5 SC 


\section{Table DR2}

date stn phase backazimuth inc phi dt method

2013.109 ABVI SKS 335.27 7.45 -49.73 0.55 RC

2013.045 ABVI SKS 350.05 9.98 -34.95 0.05 RC

2013.037 ABVI SKKS $272.1910 .76-8.814 .00$ RC

2012.356 ABVI SKKS $266.8410 .84-38.160 .03$ RC

2012.316 ABVI SKS 25.67 4.56 -24.33 0.28 RC

2011.051 ABVI SKS 335.78 9.20 -45.220 .00 RC

2011.052 ABVI SKS 229.26 5.36 45.26 0.03 2.2 RC

2012.227 ABVI SKKS $340.5812 .11-31.420 .402 .0$ RC

2013.106 ANWB SKS 50.78 7.30 -83.22 0.28 2.8 RC

2013.099 ANWB SKS 55.21 8.44 14.21 0.00 RC

2012.227 ANWB SKS 342.05 7.23 -67.95 0.38 3.4 RC

2012.224 ANWB SKS 48.09 9.72 -84.91 0.20 RC

2005.339 AOPR SKKS 93.86 12.62 42.86 0.03 RC

2005.346 AOPR SKS 35.92 6.83 42.92 0.05 RC

2005.350 AOPR SKS 334.94 6.30 47.94 0.00 RC

2006.033 AOPR SKS $258.656 .41-51.350 .400 .6$ RC

2006.102 AOPR SKS 334.439 .4840 .430 .18 RC

2006.271 AOPR SKS 258.37 7.28 45.37 0.00 RC

2011.086 AOPR SKS $334.806 .30-65.200 .482 .5$ RC

2012.224 AOPR SKS 46.46 9.18 -73.54 0.30 RC

2012.275 AOPR SKS 334.806 .4819 .800 .204 .0 RC

2013.144 CBYP SKS 338.38 8.42 -25.62 0.33 RC

2007.031 CBYP SKS 245.55 6.26 -25.45 0.35 RC

2007.008 CBYP SKS 257.836 .42 -12.17 0.50 RC

2006.219 CBYP SKS 264.04 5.06 70.04 0.45 RC

2006.033 CBYP SKS $258.836 .33-23.170 .05$ RC

2003.124 CBYP SKS 244.656 .2343 .650 .11 RC

2003.208 CBYP SKS $254.836 .52-34.170 .09$ 2.6 RC

2005.165 CBYP SKS 325.17 10.14-65.83 0.43 0.4 RC

2006.002 CBYP SKS 256.39 6.31-22.61 0.05 RC

2006.033 CBYP SKS 258.81 6.34 69.81 0.00 RC

2006.053 CBYP SKS 107.83 7.84 89.83 0.53 RC

2006.055 CBYP SKS 258.806 .2377 .800 .08 RC 
2006.057 CBYP SKS 252.65 6.06 -42.35 0.35 RC 2006.090 CBYP SKS 50.30 8.68 -56.70 0.18 RC 2008.015 CBYP SKS 254.39 6.13 76.39 0.00 RC 2008.201 CBYP SKS 259.01 6.50 -7.99 0.10 RC 2011.070 CBYP SKS 335.13 6.25 67.13 0.50 RC 2011.093 CBYP SKS 258.97 6.32 75.97 0.05 RC 2011.108 CBYP SKS 240.456 .03 -30.55 0.40 RC 2011.210 CBYP SKS 252.506 .0572 .500 .10 RC 2012.342 CBYP SKS 333.57 6.29 -3.43 0.50 RC 2013.131 CBYP SKS 257.86 6.79 -14.14 0.12 RC 2013.143 CBYP SKS 255.22 6.64 79.22 0.10 RC 2013.144 CBYP SKS 338.26 8.39 -26.74 0.40 RC 2013.274 CBYP SKS 337.45 8.17 -17.55 0.62 RC 2012.227 CDVI SKS 340.31 7.36 -52.69 0.45 RC 2006.002 CDVI SKS 256.34 6.23 -52.66 0.05 RC 2012.169 CDVI SKKS 336.67 11.59-50.33 0.50 RC 2012.176 CDVI SKS 336.47 9.37 -58.53 0.40 RC 2012.224 CDVI SKS 47.07 9.36 -74.93 0.10 RC 2012.227 CDVI SKS 340.31 7.36 -49.69 0.00 RC 2012.316 CDVI SKKS 25.6610 .5568 .66 0.20 RC 2013.028 CDVI SKS 27.38 7.01 -42.62 0.10 RC 2013.031 CDVI SKKS 270.92 10.88-44.08 0.00 RC 2014.043 CDVI SKS 29.90 6.23 65.90 0.20 2.6 RC 2013.144 CDVI SKS 338.77 8.26 -58.23 0.08 RC 2013.138 CDVI SKS 336.27 6.04-44.73 0.08 RC 2013.109 CDVI SKS 334.80 7.34 30.80 0.10 1.0 RC 2012.342 CDVI SKS 334.32 6.21 -59.68 0.40 RC 2012.270 CDVI SKS $325.1310 .22-62.87$ 0.20 0.7 RC 2012.176 CDVI SKS 336.35 9.32 -57.65 0.30 RC 2008.009 CDVI SKKS 30.26 11.39 7.26 0.80 1.0 RC 2010.216 CDVI SKKS 325.09 12.94 88.09 0.30 RC 2011.068 CDVI SKS 335.55 6.19 -55.45 0.55 2.8 RC 2011.070 CDVI SKS 335.90 6.16 32.90 0.03 RC 2011.071 CDVI SKS 335.27 6.09 -57.73 0.35 RC 2011.086 CDVI SKS 336.24 6.16 30.24 0.03 RC 
2011.093 CDVI SKS 258.956 .24 43.95 0.35 RC

2011.108 CDVI SKS 240.22 5.94 31.22 0.30 RC

2011.191 CDVI SKS 335.04 6.18 -75.96 0.60 2.2 RC

2012.227 HUMP SKS 339.69 7.45 844.0 SC

2013.106 HUMP SKS 48.47 6.96 62 4.0 SC

2013.109 HUMP SKS 334.307 .46764 .0 SC

2013.131 HUMP SKS 257.746 .78 -88 4.0 SC

2013.240 HUMP SKS 247.77 5.99 -12 4.0 SC

2013.228 MPR SKS 231.475.65 57 4.0 SC

2012.057 MPR SKS 10.97 7.37 19 4.0 SC

2007.273 MPR SKS 220.27 5.05 302.7 SC

2006.053 MPR SKS 107.61 7.71304.0 SC

2006.033 MTP SKS $258.806 .30-25.200 .10$ RC

2013.109 MTP SKS 334.36 7.41-60.64 0.33 RC

2013.131 MTP SKS 257.86 6.76 -41.14 0.12 RC

2013.143 MTP SKS 255.21 6.60 -43.79 0.08 RC

2013.144 MTP SKS $338.408 .36-51.600 .25$ RC

2012.224 OBIP SKS 46.35 9.19-84.65 0.25 RC

1993.219 SJG SKS 252.16 6.0676 4.0 SC

1994.042 SJG SKS 259.87 5.19-0 4.0 SC

1994.068 SJG SKS 258.606 .37833 .8 SC

1994.090 SJG SKS 254.21 6.17 -64 0.2 SC

1994.243 SJG SKS 335.58 6.97 -10 4.0 SC

1994.300 SJG SKS 250.056 .01824 .0 SC

1995.017 SJG SKS 255.356 .23834 .0 SC

1995.036 SJG SKS 236.34 5.94 74 4.0 SC

1995.103 SJG SKS 266.22 5.36 2 3.8 SC

1995.180 SJG SKS 259.055 .19 -89 4.0 SC

1995.184 SJG SKS 245.846 .34804 .0 SC

1995.209 SJG SKS 254.386 .69824 .0 SC

1995.279 SJG SKS 255.636 .65844 .0 SC

1995.359 SJG SKS 246.906 .41734 .0 SC

1996.218 SJG SKS 255.376 .31813 .8 SC

1996.336 SJG SKS 244.566 .06753 .8 SC

1997.080 SJG SKS 243.88 5.9976 4.0 SC 
1998.136 SJG SKS 253.896 .17803 .2 SC 1998.361 SJG SKS 253.986 .57803 .8 SC 1999.103 SJG SKS 254.226 .56824 .0 SC 1999.110 SJG SKS 242.956 .18674 .0 SC 1999.209 SJG SKS 244.676 .28754 .0 SC 2000.028 SJG SKS 334.64 6.97 71 3.8 SC 2000.166 SJG SKS 250.52 5.88 814.0 SC 2001.185 SJG SKS 253.936 .53804 .0 SC 2001.255 SJG SKS 255.196 .24814 .0 SC 2002.290 SJG SKS 256.326 .31844 .0 SC 2003.208 SJG SKS 254.626 .55814 .0 SC 2004.154 SJG SKS 241.856 .11704 .0 SC 2006.136 SJG SKS 243.346 .13713 .9 SC 2006.090 STVI SKS $245.696 .29-62.310 .23$ RC 2012.227 STVI SKS 340.23 7.43 -43.77 0.50 RC 


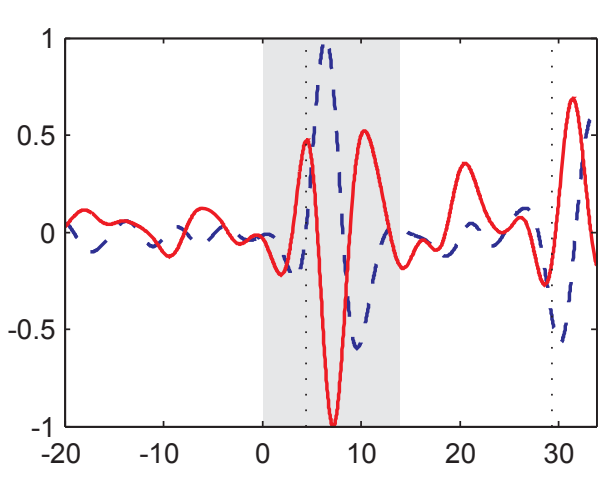

Event: 12-Nov-2013 (316) 07:03 54.68N 162.29E 47km Mw=6.5

Station: HUMP Backazimuth: 334.2 Distance:

init.Pol.: 167.8 Filter: $0.020 \mathrm{~Hz}-0.20 \mathrm{~Hz} \quad$ SNR $_{\mathrm{SC}}: 8.8$
Rotation Correlation: $-84<-67<-42 \quad 0.5<0.7 s<1.1$
Minimum Energy: $80<82<-92 \quad 1.2<1.6 s<2.1$
Eigenvalue: $\quad-88<-76<-60 \quad 0.7<0.9 \mathrm{~s}<1.2$

Quality: good IsNull: no Phase: SKS
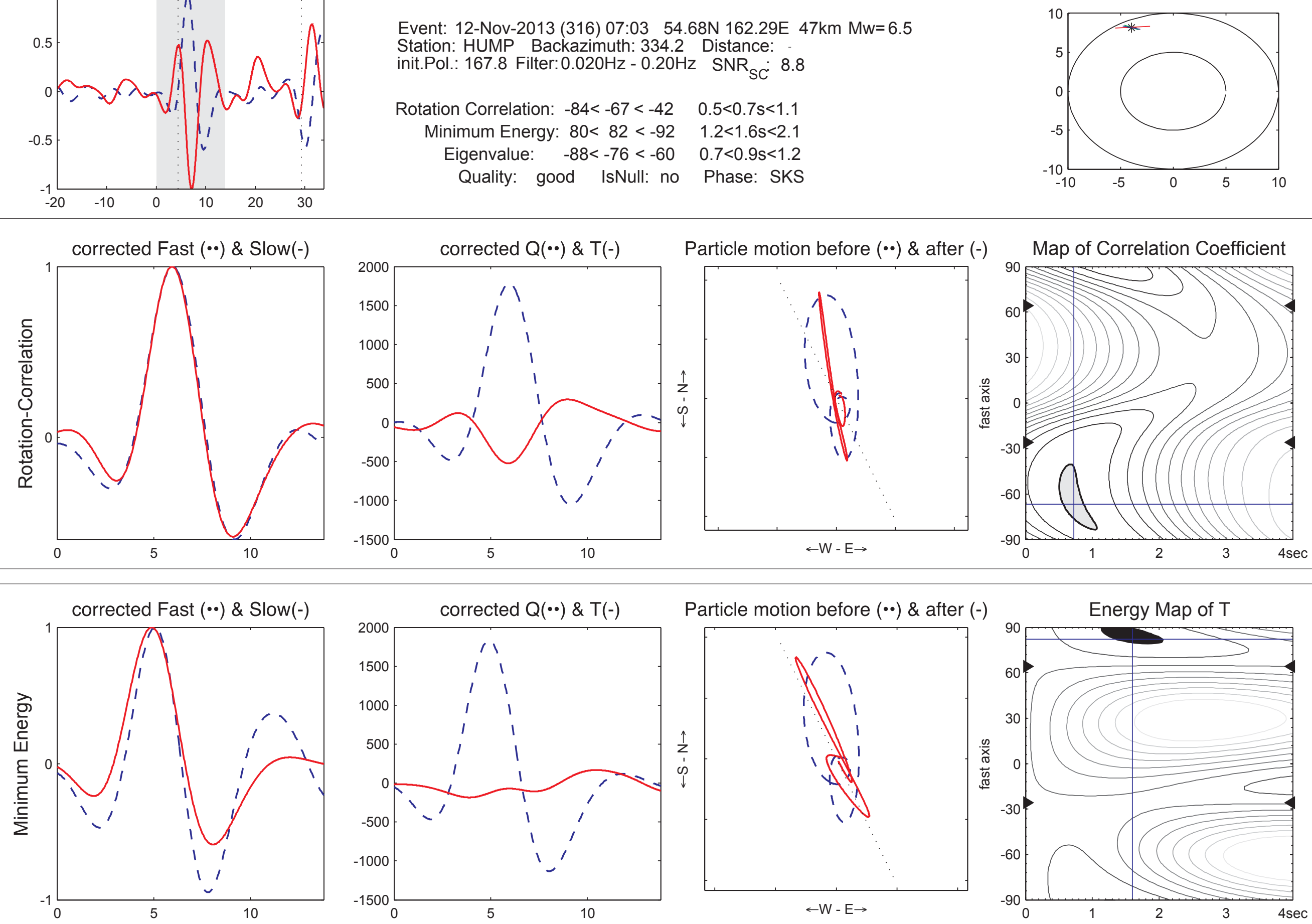